\title{
EchoGéo
}

$7 \mid 2008$

Les nouveaux enjeux régionaux dans l'océan Indien occidental

\section{La qualité des plages au cœur des enjeux de développement : La situation des îles de l'océan Indien}

(Réunion, Maurice, Seychelles)

\section{Virginie Duvat}

\section{(2) OpenEdition}

Journals

Édition électronique

URL : https://journals.openedition.org/echogeo/8993

DOI : $10.4000 /$ echogeo.8993

ISSN : 1963-1197

Éditeur

Pôle de recherche pour l'organisation et la diffusion de l'information géographique (CNRS UMR 8586)

Référence électronique

Virginie Duvat, «La qualité des plages au cœur des enjeux de développement : La situation des îles de l'océan Indien », EchoGéo [En ligne], 7 | 2008, mis en ligne le 06 février 2009, consulté le 31 juillet 2021. URL : http://journals.openedition.org/echogeo/8993 ; DOI : https://doi.org/10.4000/echogeo.8993

Ce document a été généré automatiquement le 31 juillet 2021.

EchoGéo est mis à disposition selon les termes de la licence Creative Commons Attribution - Pas d'Utilisation Commerciale - Pas de Modification 4.0 International (CC BY-NC-ND) 


\title{
La qualité des plages au cœur des enjeux de développement : La situation des îles de l'océan Indien
}

\author{
(Réunion, Maurice, Seychelles)
}

\section{Virginie Duvat}

1 Dans nombre de pays continentaux (États-Unis, Afrique du Sud, Royaume-Uni, Brésil, en particulier) et d'îles tropicales (Iles Vierges Américaines, Hawaii, par exemple), la question de la qualité des plages est devenue une préoccupation majeure à partir des décennies 1970 et 1980, parallèlement au développement du tourisme. Dans les îles de l'ouest de l'océan Indien dont la mise en tourisme date du début des années 1970, elle s'est progressivement imposée dans les politiques de gestion du littoral au cours des deux dernières décennies pour trois raisons principales. La première est économique, les côtes sableuses constituant le support privilégié du tourisme dont le rôle dans le processus de développement a été majeur (Magnan, 2005). La deuxième est environnementale, les acteurs publics mettant en place des stratégies visant à réduire les impacts destructeurs des activités humaines sur les écosystèmes récifaux (Duvat, 2007 ; Cazes-Duvat et Paskoff, 2004). En dernier lieu, l'intérêt croissant des autorités pour la qualité des plages tient aussi à des facteurs sociétaux, l'augmentation du niveau de vie ayant soutenu l'émergence des pratiques récréatives et touristiques des résidents. À Maurice, l'aménagement des plages publiques et l'ouverture de centres nautiques destinés à la population traduisent bien la profonde mutation socioéconomique et culturelle qui opère sur le littoral (Magnan, 2007).

2 C'est bien parce qu'elle s'inscrit au cœur d'enjeux majeurs que la qualité des plages, qui n'avait au départ été mobilisée dans les discours officiels que dans le but de promouvoir le tourisme (Gay, 2004 ; Duvat, à paraitre), est récemment entrée dans les politiques de gestion des côtes ${ }^{1}$. L'intervention des pouvoirs publics en faveur de la qualité du littoral prend désormais trois formes. La première est la création d'espaces protégés et, en particulier, de parcs marins (dès 1973 aux Seychelles, entre 1997-2007 à la Réunion et à partir de 1999 à Maurice). La seconde est la mise en place d'une politique foncière qui 
vise à limiter l'urbanisation et, par là même, à maintenir le libre accès du public aux rivages ${ }^{2}$. Cette politique est portée par le Conservatoire du littoral à la Réunion (Klein, 2003) et par le Ministère du logement et des terres à l'île Maurice ${ }^{3}$. Pour compléter ce dispositif, ont été créées des structures chargées de l'entretien du littoral, la SEM balnéaire de Saint-Gilles à la Réunion ${ }^{4}$ et la Beach Authority (2002) à Maurice, qui conduisent des actions variées (nettoyage, aménagement pour le public, travaux paysagers, défense légère des côtes).

3 La mise au point d'outils d'évaluation et de suivi de la qualité des plages intéresse aujourd'hui les responsables politiques et administratifs des petits États insulaires et des îles françaises d'outre-mer. C'est pour répondre à cette demande que l'étude dont les résultats sont ici présentés a été réalisée. Ces outils permettent, premièrement, de mesurer les impacts des aménagements et des pratiques touristiques sur l'environnement; deuxièmement, d'évaluer l'efficacité des politiques d'aménagement et de gestion, qui visent en particulier à réduire les dégradations environnementales et à améliorer la qualité de l'accueil ; troisièmement, de constituer des bases de données dans une perspective d'aide à la décision.

Cet article abordera d'abord les questionnements méthodologiques que soulève une telle démarche. Quels critères retenir pour évaluer la qualité des plages? Comment les hiérarchiser ? Il présentera ensuite la méthodologie et les résultats de l'évaluation qui a été effectuée dans plusieurs îles de l'océan Indien. Cela permettra de répondre aux questions suivantes : la réputation des plages tropicales se fonde-t-elle sur des attributs spécifiques? Tient-elle principalement aux facteurs naturels qui définissent la tropicalité ? En dernier lieu, il s'agira de voir dans quelle mesure la qualité des plages renvoie aux stratégies de développement des différents acteurs qui interviennent sur le littoral, non seulement publics, mais aussi privés.

\section{Aspects méthodologiques}

On s'emploiera ici à dresser un état des lieux des travaux antérieurs afin de poser les bases conceptuelles et méthodologiques d'une évaluation de la qualité des plages.

\section{La qualité des plages, une question qui s'inscrit à l'interface science/gestion/société}

6 Au cours des deux dernières décennies, la question de la qualité des plages a changé de statut. Elle a d'abord constitué un objet d'étude et de préoccupation pour les spécialistes des questions environnementales qui se sont alarmés des dégradations qu'ils ont pu observer. Dans un deuxième temps, elle a été prise en charge par les gestionnaires, avant de devenir, très récemment, une question de société sous l'effet de divers processus (lancement des politiques de sensibilisation et de responsabilisation des usagers, prise en compte de leurs attentes). Cette évolution, qui la place résolument au cœur des politiques de gestion intégrée du littoral, a considérablement fait évoluer les méthodologies d'évaluation.

7 Les premiers travaux ont porté sur la qualité de l'environnement littoral, appréhendé à travers ses différentes composantes (récifs coralliens, eaux côtières, plages...). Ils ont été réalisés sur la base de critères proposés par des scientifiques dans le but de dresser 
des diagnostics, aussi reposent-ils sur des indicateurs d'état élaborés à partir de données physiques, biologiques et biochimiques directement collectées sur le terrain (Morgan, 1999 ; Cendrero et Fischer, 1997 ; Leatherman, 1997).

8 Les politiques supranationales, nationales et régionales ont contribué à définir la qualité des milieux à travers la fixation de normes réglementaires, comme l'illustrent bien les directives européennes relatives à la qualité des eaux de baignade. Or il apparaît que ces normes et qu'ainsi les modalités de contrôle qui leur sont associées varient suivant les pays. Les exigences des Pays-Bas sur la qualité des eaux de baignade sont par exemple supérieures à celles de l'Afrique du Sud (Morgan, 1999). On retrouve les mêmes écarts en ce qui concerne la gestion des déchets. Parce qu'elles définissent des objectifs de qualité, ces normes influent d'une part, sur les évaluations scientifiques (fixation de seuils de référence), et d'autre part, sur l'appréciation du public (apparition de références collectives). La qualité des milieux ne saurait donc être définie sans tenir compte du regard des acteurs du territoire considéré et des politiques qu'ils mènent. La complémentarité des scientifiques et des gestionnaires dans la mise en place de seuils de qualité explique que leurs points de vue soient associés dans de nombreuses évaluations (Williams et Morgan, 1995 ; Morgan, op. cit. ; Ferrarini et al., 2001 ; Ergin et al., 2008).

9 À partir des années 1990, les préférences et les attentes du public ont été intégrées dans la plupart des évaluations sur la base des propositions méthodologiques formulées par les chercheurs de l'Université du Pays de Galles. Les travaux disponibles sur l'évaluation de la qualité paysagère montraient déjà que son appréciation dépend de facteurs culturels, sociétaux, socioéconomiques, ainsi que de l'expérience individuelle des usagers (Fines, 1968 ; Zube et Pitt, 1981 ; Kaplan et Kaplan, 1989). L'intégration des attentes du public paraît d'autant plus importante dans les îles tropicales qu'elles accueillent sur leurs littoraux des catégories de population variées, la diversité des clientèles extérieures s'ajoutant parfois au caractère multiethnique de la population. Ainsi, l'étude pionnière qui a été réalisée dans les Iles Vierges Américaines dans les années 1970 a montré que si les touristes occidentaux apprécient tout particulièrement les côtes non urbanisées, ce n'est en revanche pas le cas des résidents qui assimilent le bâti au développement et à l'apparition de revenus (Virgin Islands Planning Office, 1977).

10 La notion de qualité renvoie nécessairement à un contexte sociétal, socioéconomique et politique. La qualité est relative à des normes établies par la société et à des perceptions qui évoluent en permanence. Cela justifie d'intégrer l'ensemble des acteurs et des usagers dans son évaluation, comme l'expriment A. Cendrero et D.W. Fischer (1997) : "Any attempt at establishing a system to determine and compare levels of environmental quality must take into consideration human opinions and preferences, be it from scientists, planners, decision-makers, specific stakeholder groups or the general public ».

\section{Les fondements méthodologiques des travaux d'évaluation}

11 Les méthodologies d'évaluation qui sont aujourd'hui utilisées reposent sur l'identification, la validation et la hiérarchisation des critères de qualité, à partir de la consultation de gestionnaires, de scientifiques et d'usagers sur la base d'entretiens et d'enquêtes. Ces études associent deux démarches: un travail de caractérisation des plages - fondé sur des relevés de terrain, des données issues de travaux antérieurs et de 
documents d'archives, des évaluations quantitatives et qualitatives de différents paramètres et sur l'exploitation d'images aériennes - et la réalisation d'enquêtes auprès du public.

La première démarche vise à déterminer les attributs des plages et à les synthétiser dans des tableaux de bord utilisables par les gestionnaires. Comme on l'a vu, elle repose sur des bases scientifiques et réglementaires (rôle des normes dans l'évaluation de l'état observé). Parce qu'elle permet de connaître les préférences des usagers, la seconde sert à établir la hiérarchie des facteurs d'attractivité et à définir pour chacun d'eux des niveaux de qualité, en général cinq (Williams et Morgan, 1995). Ainsi, les systèmes d'évaluation associent deux types de données: des données d'état qui permettent de qualifier l' "objective beach" (Pereira et al., 2003) ou, ce qui revient au même, de calculer un "Beach Index» basé sur des données descriptives (Cervantes et Espejel, 2008), et des données subjectives qui définissent la «subjective beach» ou le "Knowledge Index », fondés sur la perception des usagers. Suivant les cas, les politiques de qualité privilégient les premières (aires protégées, par ex.) ou les secondes (littoraux à vocation touristique, par ex.).

Les études de qualité sont conduites à des échelles variées, de l'échelon régional à l'échelon local. Les travaux qui portent sur les plages privilégient la grande échelle, celle de la plage, qui constitue une unité cohérente à la fois sur le plan physique (cellule ou sous-cellule morpho-sédimentaire) et en termes de gestion. Suivant les cas, l'évaluation porte sur la plage prise dans sa globalité ou sur des segments clairement identifiés. Ainsi, R. Morgan (1999) distingue deux situations, celle des plages de poche ou des plages urbaines dont la petite dimension et l'unité invitent à réaliser une évaluation globale, et celle des plages plus vastes. Dans ce cas, il propose de faire porter l'évaluation sur la partie centrale de la plage parce qu'elle concentre la fréquentation et les efforts de gestion. Il est également possible de découper les plages en segments homogènes à partir de leurs caractéristiques physiques et humaines afin de pouvoir les évaluer sur toute leur longueur et de mettre en évidence leur diversité (Duvat, 2008).

Enfin, la variabilité des caractéristiques physiques et biologiques que présentent les plages (Cendrero et al., 2003) peut être appréhendée de deux manières en fonction de la durée de la saison touristique. Là où celle-ci est brève, une seule évaluation suffit à caractériser la qualité des plages (Morgan, 1999). En revanche, il est souhaitable de réaliser plusieurs évaluations dans l'année lorsque la qualité des plages varie au cours de la saison touristique, à moins que ces variations ne soient corrélées à des phénomènes exceptionnels (cyclones en milieu tropical, par ex.).

\section{Les facteurs d'attractivité des plages}

15 Quatre facteurs d'attractivité ressortent des différentes études qui ont été conduites à travers le monde (Morgan et Williams, 1995 ; Leatherman, 1997; Nelson et al., 2000 ; Deng et al., 2002; Micallef, 2002) : la qualité du paysage, qui joue en général un rôle majeur; la qualité sanitaire (eau et déchets), à laquelle le public est de plus en plus sensible, mais qu'il apprécie principalement à partir de critères visuels en dépit des mesures de qualité des eaux qui sont réalisées; la sécurité, en particulier de la baignade, qui explique la forte fréquentation des plages surveillées; le niveau d'aménagement et d'équipement, qui détermine le confort et la capacité récréative des sites. D'autres facteurs jouent un rôle variable suivant les contextes, comme le 
patrimoine local, naturel et culturel, et les usages locaux (Yang, 1994 ; Ethos Consulting, 1991 ; Deng et al., 2002 ; Micallef, 2002).

L'importance relative des facteurs naturels et anthropiques dans l'appréciation de la qualité des plages varie selon les types de publics, les clientèles urbaines étant plus attachées aux équipements et aux services que les populations d'origine rurale qui manifestent en revanche un fort intérêt pour les attributs "naturels" (végétation, absence de bâti...). Ce constat donne toute leur pertinence aux travaux qui distinguent les plages urbaines des plages rurales ou "sauvages». A. Micallef et al. (2004) définissent jusqu'à cinq types de plages qui répondent à des attentes différentes, les plages d'hôtels, les plages urbaines, les plages situées à proximité d'un village, les plages rurales et les plages isolées.

Suivant les cas, le nombre de critères varie d'une vingtaine à une cinquantaine. Le poids relatif des différents critères et des grandes catégories de facteurs (physique, biologique, humain...) dépend des contextes et des objectifs visés par l'étude.

Sur ces bases, quelle méthodologie d'évaluation appliquer dans les petites îles touristiques de l'ouest de l'océan Indien? Quelles spécificités de ces espaces doivent être prises en compte pour l'élaboration d'une trame méthodologique adaptée?

\section{Évaluation de la qualité des plages des seychelles et des mascareignes}

Il s'agira ici de présenter rapidement le contexte local, puis d'exposer la méthodologie qui a été appliquée ainsi que les principaux résultats qu'elle a permis de produire.

\section{Principales caractéristiques des plages et des clientèles touristiques}

Dans les deux archipels qui nous concernent, les plages de sable sont d'origine corallienne ; à la Réunion, s'y ajoutent quelques plages de sable noir basaltique (CazesDuvat, 1999; Cazes-Duvat et Paskoff, 2004). Les plages de poche alternent avec des plages de fond de baie et avec de grandes plages rectilignes qui s'étirent sur quelques kilomètres. La largeur des plages varie de quelques mètres à plus de $50 \mathrm{~m}$. Leur pente, leur granulométrie (présence de débris et blocs de corail, en particulier) et leur fonctionnement morphodynamique (existence ou non d'un déferlement), varient en fonction de la présence et de la dimension des récifs coralliens. Ceux-ci déterminent également les conditions de baignade, très mauvaises là où le platier est continu et affleurant. Certaines plages de la Réunion et de Maurice sont adossées à un massif dunaire alors que d'autres sont en contact direct avec des zones urbaines et des hôtels. Aux Seychelles, les plages sont le plus souvent bordées par une mangrove ou par une plaine littorale, ou alors directement adossées à des chaos de blocs. Elles sont nettement moins marquées par l'urbanisation que celles des Mascareignes. Situées en milieu microtidal $(0,60 \mathrm{~m}$ de marnage moyen à Maurice et à la Réunion contre $1,20 \mathrm{~m}$ aux Seychelles), l'ensemble de ces plages offrent des conditions de pratique relativement stables à l'échelle d'une journée. Les plages des îles tropicales se distinguent de celles des façades continentales de la zone tempérée par leur ambiance climatique (chaleur et souvent humidité), par leurs paysages (végétation dense et 
couleurs vives) et par un environnement hyperocéanique (vue sur la ligne d'horizon, sur le large ou sur des îlots). Ces facteurs jouent un rôle central dans l'attraction des touristes en participant au processus de mythification (Cazes, 1989).

Dans ces îles, les plages d'hôtels et celles des stations balnéaires sont principalement fréquentées par des touristes dont l'origine est essentiellement occidentale (80\% d'Européens). Les autres plages, qualifiées de "publiques " à Maurice, accueillent une majorité de résidents dont les activités sont pour partie proches de celles des touristes (baignade, jeux de plage, surf...) et pour partie spécifiques (pique-nique créole, en particulier). Les pratiques récréatives et touristiques de la population locale ont connu un important essor au cours des dernières années, en particulier à la Réunion et à Maurice où la fréquentation des littoraux augmente continuellement ${ }^{5}$.

Dans les Mascareignes, le processus de développement qui a marqué les quatre dernières décennies est très influencé par le modèle occidental, ce qui explique que le système balnéaire européen soit devenu une référence, d'abord à la Réunion où l'influence des Métropolitains s'exerce depuis la départementalisation (1946), puis à l'île Maurice où l'essor du tourisme a eu pour effet de faire évoluer les pratiques littorales (développement des pratiques balnéaires, des sports de glisse et du nautisme). Dans ce contexte, touristes et résidents partagent diverses attentes, qui concernent en particulier la sécurité (de la baignade, des personnes et des biens) et le confort (granulométrie, couleur du sable ${ }^{6}$, équipements élémentaires...).

Aux Seychelles où l'influence occidentale reste réduite (Gay, 2004), la fréquentation des plages par les résidents est modérée.

\section{Grille méthodologique}

Le caractère relativement homogène des pratiques et des attentes des différentes catégories d'usagers, établi par la réalisation d'observations de terrain et d'enquêtes, a conduit à élaborer une grille d'évaluation unique pour les touristes extérieurs et les résidents. Par ailleurs, au regard de la faible diversité des plages (absence de véritables plages « urbaines » et degré d'équipement relativement réduit de la plupart des plages "publiques»), il n'a pas paru nécessaire de distinguer ces deux catégories. La principale opposition qui s'observe est à Maurice celle des plages d'hôtels et des plages publiques. Elle n'a pas donné lieu à la mise en place de deux grilles d'évaluation distinctes afin que les résultats produits dans les différentes îles puissent être comparés.

L'évaluation a été réalisée sur la base de 40 critères comportant chacun 5 niveaux de qualité qui ont été déterminés à partir de la réalisation d'une enquête auprès des usagers (tab. 1). Ces critères se répartissent de la manière suivante: 10 sont d'ordre physique, 8 sont d'ordre biologique, 15 sont relatifs à des aspects humains et 7 résultent de la combinaison de facteurs physiques et anthropiques. Ils couvrent la qualité de l'environnement et de l'accueil, et une place non négligeable est attribuée au paysage. 
Tableau 1 - Grille d'évaluation de la qualité des plages

\begin{tabular}{|c|c|c|c|c|c|}
\hline \multirow[t]{2}{*}{ Critères de qualité } & Niveau 1 & Niveau 2 & Niveau 3 & Niveau 4 & Niveau 5 \\
\hline & $<5 m$ & $5.10 \mathrm{~m}$ & $10-15 \mathrm{~m}$ & $15-20 \mathrm{~m}$ & $>20 \mathrm{~m}$ \\
\hline \multirow{31}{*}{ 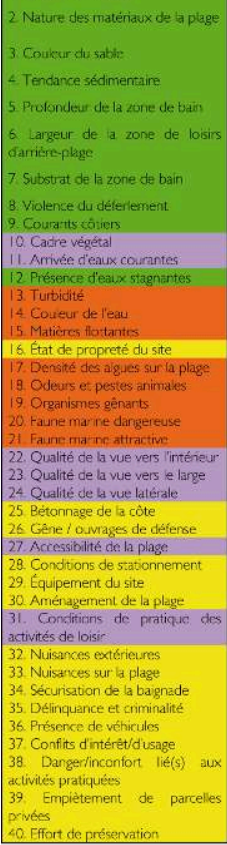 } & Galets & Nbx débris & Qqs débris + sable & Sable grossier & à \\
\hline & Gris & Noir & Cosdeur teme & Bance & $\begin{array}{l}\text { Blanc ou rose } \\
\text { prllart }\end{array}$ \\
\hline & Recul importare & Comblement & Recul notable & Fable recul & Stabilité relative \\
\hline & Importante & Tress laible & $0.50 \mathrm{mà} I \mathrm{~m}$ & Pente raide. & $\begin{array}{l}\text { Pente moyenne, } \\
\text { prof. }>1 \mathrm{~m}\end{array}$ \\
\hline & $<5 \mathrm{~m}$ & $5-10 \mathrm{~m}$ & $10-20 \mathrm{~m}$ & $20.50 \mathrm{~m}$ & $>50 \mathrm{~m}$ \\
\hline & RocheriBR/Galets & BR massifiplatier & $\begin{array}{l}\text { Herbier ettou } \\
\text { débris }\end{array}$ & Sable et débris & Sable \\
\hline & $\begin{array}{l}\text { Très elevée } \\
\text { Forts permarents }\end{array}$ & $\begin{array}{l}\text { Ellevée } \\
\text { Forts saisorniturs }\end{array}$ & $\begin{array}{l}\text { Moyenne } \\
\text { Forts ponctuels }\end{array}$ & $\begin{array}{l}\text { Fable } \\
\text { Faables }\end{array}$ & $\begin{array}{c}\text { Nulle } \\
\text { Absents }\end{array}$ \\
\hline & $\begin{array}{l}\text { Très dégrade } \\
\text { Embouchure }\end{array}$ & $\begin{array}{l}\text { Absent } \\
\text { Rivos centrale }\end{array}$ & $\begin{array}{l}\text { Qualté noyerne } \\
\text { Rvy extr de phase }\end{array}$ & $\begin{array}{l}\text { Bonne qualité } \\
\text { Resurgerces }\end{array}$ & $\begin{array}{l}\text { T. bonne qualite } \\
\text { Aucune. }\end{array}$ \\
\hline & & Saison & Temp & & \\
\hline & $\begin{array}{l}\text { Très śt } \\
\text { Noit }\end{array}$ & & $\begin{array}{l}\text { Moyerne } \\
\text { Beu ou wert-gis }\end{array}$ & & \\
\hline & Très frante densité & Forted d & Densité mayenre & & \\
\hline & $\begin{array}{l}\text { Très insuffisart: } \\
\text { Trés slevée }\end{array}$ & $\begin{array}{l}\text { Insuffisant } \\
\text { Elevere } \\
\text { Elate }\end{array}$ & $\begin{array}{l}\text { Moyen } \\
\text { Moyenne }\end{array}$ & $\begin{array}{l}\text { Comect } \\
\text { Fable }\end{array}$ & $\begin{array}{l}\text { Satisfaisant } \\
\text { Nulle }\end{array}$ \\
\hline & $\begin{array}{l}\text { Fortes pemarentes } \\
\text { Permanants }\end{array}$ & $\begin{array}{l}\text { Fortes saisornières } \\
\text { Sticonniers }\end{array}$ & $\begin{array}{l}\text { Temporaires } \\
\text { Temporires }\end{array}$ & $\begin{array}{l}\text { Rares } \\
\text { Rares }\end{array}$ & $\begin{array}{l}\text { Absentes } \\
\text { Atrents }\end{array}$ \\
\hline & $\begin{array}{l}\text { Permanents } \\
\text { Pemanentie }\end{array}$ & $\begin{array}{l}\text { Salsoni } \\
\text { Saisont }\end{array}$ & & $\begin{array}{l}\text { Rares } \\
\text { Pare }\end{array}$ & $\begin{array}{l}\text { Abssents } \\
\text { Absente }\end{array}$ \\
\hline & $\begin{array}{c}\text { Abss } \\
\text { Triss }\end{array}$ & & & & $\begin{array}{l}\text { Pormancente } \\
\text { Excellente }\end{array}$ \\
\hline & & & & & \\
\hline & & & & & \\
\hline & & Important: & Mor & & \\
\hline & Trés Elevere & & & table & \\
\hline & 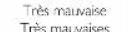 & & & $\begin{array}{l}\text { Bonne } \\
\text { Bonnes }\end{array}$ & \\
\hline & $\begin{array}{l}\text { Mresen } \\
\text { Trés in }\end{array}$ & & & $\begin{array}{l}\text { Bonnes } \\
\text { Bon }\end{array}$ & \\
\hline & Trés insulfisan: & Insulfisant & & & \\
\hline & Trìs matvaises & Maunaises & Moyennes & Bonnes & Excellentes \\
\hline & Très élevées & Élevées & Moyennes & Restuites & Nulles \\
\hline & $\begin{array}{l}\text { Treses éevives } \\
\text { Núle }\end{array}$ & $\begin{array}{c}\text { Elevéses } \\
\text { insuffisante }\end{array}$ & & & $\begin{array}{c}\text { Nulles } \\
\text { Satistasante }\end{array}$ \\
\hline & Trés flevere & & & Limitée & \\
\hline & & & & Services & \\
\hline & Très ît & & Moye & & \\
\hline & Très ślewé & Elevé & Moyen & Fable & Nul \\
\hline & Sur tout le linéaire & $>$ motié du linéare & $1 / 3$ à $1 / 2$ du linéaire & $<1 / 3$ & Nul \\
\hline & $\mathrm{Nu}$ & Très rédut & Moyen & Assez important & Important \\
\hline
\end{tabular}

Le patrimoine culturel n'a pas été intégré dans la grille d'évaluation parce qu'étant peu présent sur le littoral, il joue un faible rôle dans l'attractivité des plages.

\section{Des plages de qualité inégale}

La longueur du linéaire sableux des îles étudiées ${ }^{7}$ rendait difficile la réalisation d'un travail exhaustif. Le choix de l'échantillon a été effectué sur la base de deux critères, le niveau de fréquentation des plages (qui traduit leur attractivité et rend nécessaire leur gestion) et leur diversité (plages de sable blanc et de sable noir à la Réunion; plages "publiques" et plages d'hôtel à Maurice). Les 44 plages qui ont été évaluées se répartissent entre les différentes façades des îles étudiées ${ }^{8}$. Sur ces bases, l'échantillon choisi est tout à fait représentatif de la diversité que l'on rencontre dans ces deux archipels.

Le taux d'attractivité des plages considérées varie de 57 à $87,5 \%$ (tab. 2 et 3). Les valeurs les plus basses $(<65 \%)$ correspondent à des plages situées dans des zones urbanisées au plus près de la mer et/ou dénuées de qualités naturelles (baignade impraticable, accumulation de galets de corail, présence de dalles de beachrock ${ }^{9}$, très forte érosion...) (planche 1). Ces plages sont peu fréquentées, seulement par des familles qui résident à proximité, et elles ne bénéficient d'aucune intervention publique visant à améliorer leur qualité. On les trouve en général dans les zones urbanisées (Maurice) et au pied de routes côtières (La Réunion, Seychelles).

Les plus attractives (> $80 \%$ ) sont les plages des hôtels de grand standing et les plages "mythiques» (planche 1). Nombreuses à l'île Maurice, les premières présentent de multiples atouts. Leurs qualités «naturelles » ont souvent été améliorées par diverses interventions (rechargement artificiel en sable, végétalisation) et elles présentent un 
haut niveau d'équipement. Les plages "de rêve» ou "mythiques" sont bien représentées aux Seychelles où plusieurs comptent parmi les plus réputées du monde pour leurs attraits naturels (Lazio à Praslin, Intendance à Mahé, Grande Anse, Petite Anse et Anse Cocos à la Digue). Le caractère exceptionnel des paysages (chaos de blocs de granite rose) que les touristes disent vouloir voir au moins une fois dans leur vie n'est pas leur seul atout. Certaines d'entre elles (anse Lazio, par exemple) offrent des conditions optimales pour les pratiques balnéaires: une végétation dense qui procure de l'ombre, un sable magnifique et des eaux turquoises, calmes et peuplées de poissons colorés. D'autres plages de cette catégorie, situées dans le sud des îles, sont dangereuses entre mai et octobre en raison d'un fort hydrodynamisme qui est à l'origine de puissants courants d'arrachement.

De nombreuses plages peuvent être qualifiées de "confortables", avec un taux d'attractivité compris entre 75 et $80 \%$. Il s'agit dans certains cas de plages touristiques qui bordent des hôtels (à Maurice et aux Seychelles) ou des stations balnéaires (Boucan Canot, Étang-Salé, Saint-Leu, Saint-Pierre à la Réunion, Pereybère à Maurice, Beau Vallon à Mahé). Dans d'autres cas, ce sont des plages isolées (Petite Anse et Anse Fourmis aux Seychelles), qui présentent des taux d'attractivité comparables en raison de leurs qualités naturelles bien qu'elles soient dépourvues d'équipements.

D'autres plages ont une attractivité moyenne (65 à $75 \%$ ). Les unes présentent de fortes contraintes physiques : baignade impossible ou difficile, nuisances liées à l'érosion côtière (ouvrages de défense dégradés, chute d'arbres, caractère massif du beachrock). Les autres sont situées dans un environnement urbain qui a des effets contrastés sur leur qualité (pollution et emprise du bâti d'un côté, mais équipements et services de l'autre) ou bordent des routes côtières.

Tableau 2 - La qualité des principales plages des Mascareignes (Réunion et Maurice)

\begin{tabular}{|r|c|c|r|c|c|}
\hline & \multicolumn{2}{|c|}{ Indice de qualité } & & \multicolumn{2}{|c|}{ Indice de qualité } \\
\hline La Réunion & Valeur & $\%$ & Maurice & Valeur & $\%$ \\
Boucan Canot I & 161 & 80,5 & Grand Baie (plage publ.) & 122 & 61 \\
Boucan Canot 2 & 129 & 64,5 & Mon Choisy (plage publ.) & 167 & 83,5 \\
Boucan Canot 3 & 153 & 76,5 & Flic en Flac nord (hôtel) & 162 & 81 \\
Cap Homard 1 & 148 & 74 & Flic en Flac (plage publ.) & 146 & 73 \\
Cap Homard 2 & 125 & 62,5 & Flic en Flac sud (hôtels) & 173 & 86,5 \\
Aigrettes & 114 & 57 & Le Morne nord (hôtels) & 156 & 78 \\
Roches Noires & 138 & 69 & Le Morne (plage publ.) & 150 & 75 \\
Brisants & 144 & 72 & Bel Ombre & 150 & 75 \\
Ermitage & 149 & 74,5 & Pomponette (plage publ.) & 163 & 81,5 \\
Saline & 143 & 71,5 & Gris Gris (plage publ.) & 157 & 78,5 \\
Trou d'Eau & 140 & 70 & La Cambuse (plage publ.) & 161 & 80,5 \\
Saint-Leu centre & 139 & 69,5 & Trou aux Biches (hôtels) & 175 & 87,5 \\
Saint-Leu sud & 160 & 80 & Ile aux Cerfs (hôtels) & 172 & 86 \\
Saint-Leu cimetière & 125 & 62,5 & Belle Mare (plage publ.) & 166 & 83 \\
Étang Salé nord & 151 & 75,5 & Belle Mare (hôtels) & 167 & 83,5 \\
Étang Salé centre & 156 & 78 & Pointe de Flacq (hôtel) & 161 & 80,5 \\
Ravine Blanche & 152 & 76 & Legends (hôtel) & 139 & 69,5 \\
Saint-Pierre centre & 148 & 74 & Pereybère (plage publ.) & 155 & 77,5 \\
& & & La Curvette (plage publ.) & 158 & 79 \\
\hline
\end{tabular}


Tableau 3 - La qualité des principales plages des Seychelles granitiques

\begin{tabular}{|c|c|c|c|c|c|}
\hline & \multicolumn{2}{|c|}{ Indice de qualité } & \multicolumn{2}{c|}{ Indice de qualité } \\
\hline La Digue & Valeur & $\%$ & Praslin & Valeur & $\%$ \\
Anse Cocos & 147 & 73,5 & Petite Kerlan (hôtel) & 167 & 83,5 \\
Petite Anse & 155 & 77,5 & Kerlan & 156 & 78 \\
Grande Anse & 172 & 86 & Lazio & 174 & 87 \\
Anse Réunion & 140 & 70 & Grand Anse & 131 & 65,5 \\
AnseUnion & 126 & 63 & Volbert ouest (hôtels) & 156 & 78 \\
Anse Source d'Argent & 169 & 84,5 & & & \\
Anse Gaulettes & 135 & 67,5 & Mahé & & \\
Anse Grosse Roche & 145 & 72,5 & Beau Vallon & 157 & 78,5 \\
Anse Banane & 140 & 70 & Grande Anse & 155 & 77,5 \\
Anse Fourmis & 150 & 75 & Anse Barbarons(hôtel) & 166 & 83 \\
Anse Sévère & 149 & 74,5 & Anse Boileau & 131 & 65,5 \\
& & & Anse Takamaka & 148 & 74 \\
& & & Anse Intendance (hôtel) & 169 & 84,5 \\
& & & Anse Marie-Louise & 145 & 72,5 \\
& & & Anse Forbans (hôtel) & 153 & 76,5 \\
& & Anse Royale & 144 & 72 \\
& & Anse Nord-Est & 141 & 70,5 \\
\hline
\end{tabular}

La comparaison des résultats obtenus dans les différentes îles fait apparaître la nette supériorité des plages mauriciennes et seychelloises dont la réputation est donc tout à fait fondée.

Plage d'anse Lazio, Praslin, Seychelles (attractivité $87 \%$ )

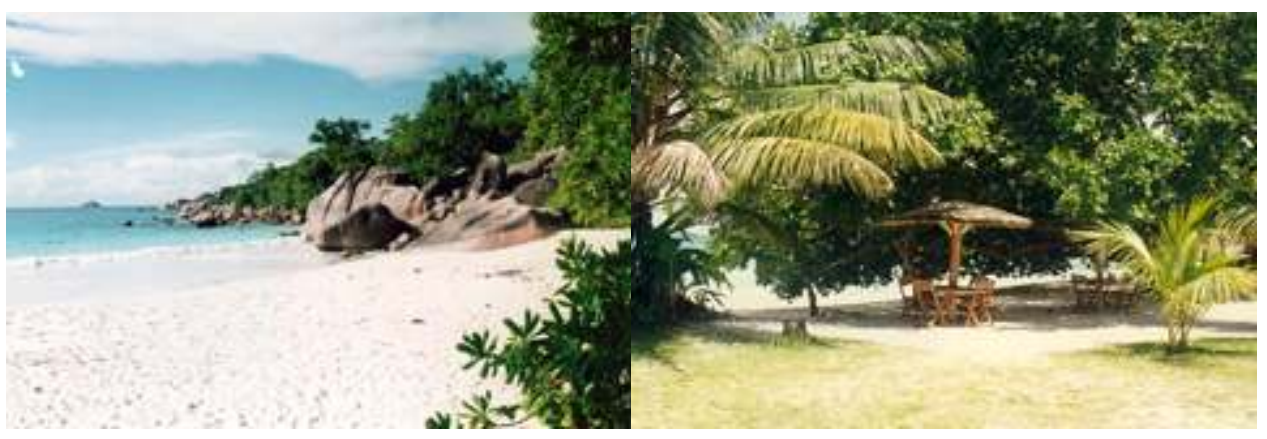

Cette plage possède tous les attributs des plages mythiques des Seychelles: sable blanc très fin, granites roses, eaux turquoises calmes et peuplées de poissons colorés, végétation foisonnante. Elle est équipée d'une aire de stationnement et possède un restaurant privé. 
Plage de Pomponnette, sud de l'île Maurice (taux d'attractivité : 81,5\%)
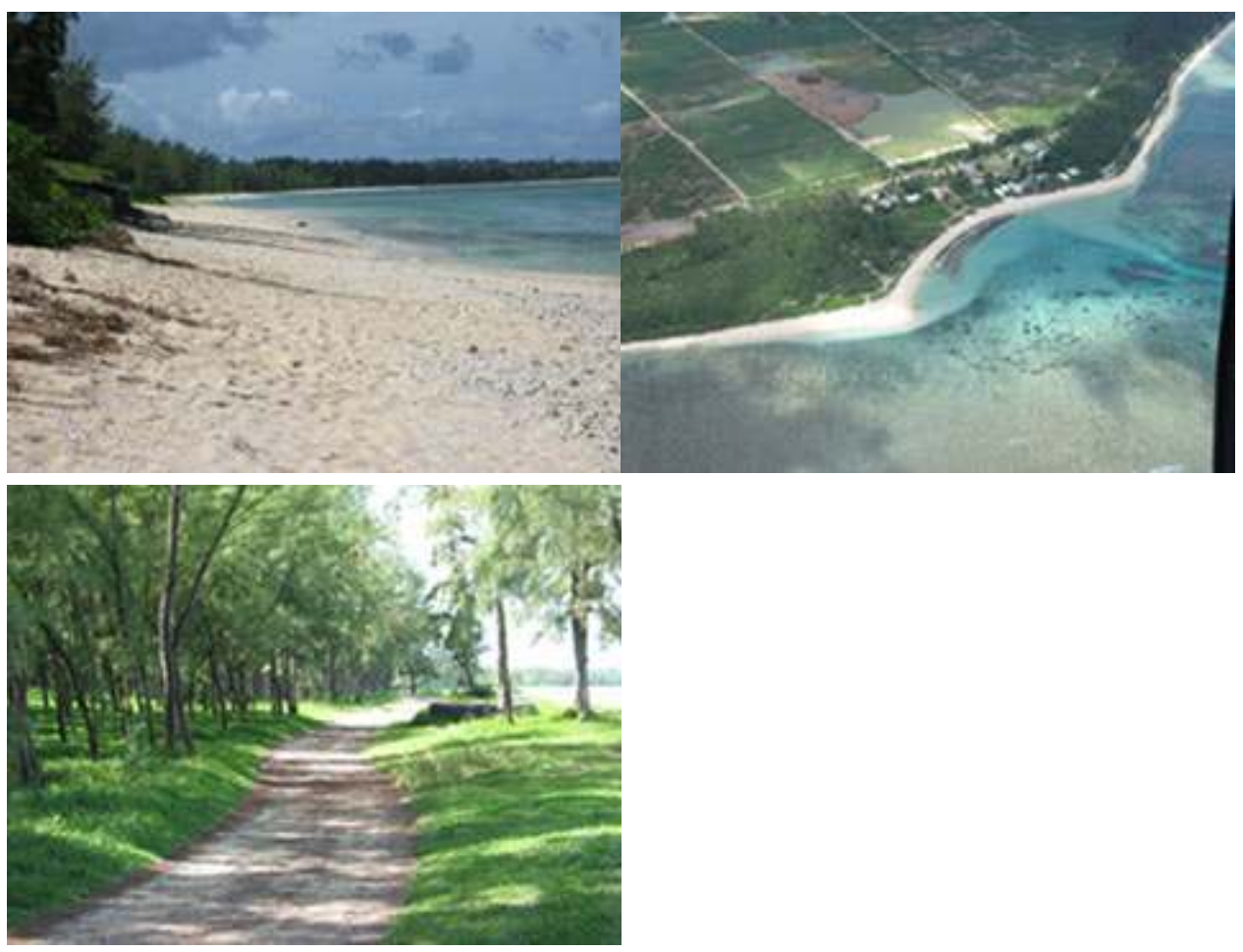

Cette plage offre des eaux calmes parce qu'elle est bordée par un récif frangeant. Elle est large d'une vingtaine de mètres et bordée de dunes qui constituent un espace de pique-nique prisé le week-end. Elle ne possède pas d'équipement spécifique

Plage de l'Ermitage, ouest de l'île de La Réunion (taux d'attractivité : 74,5 \%)

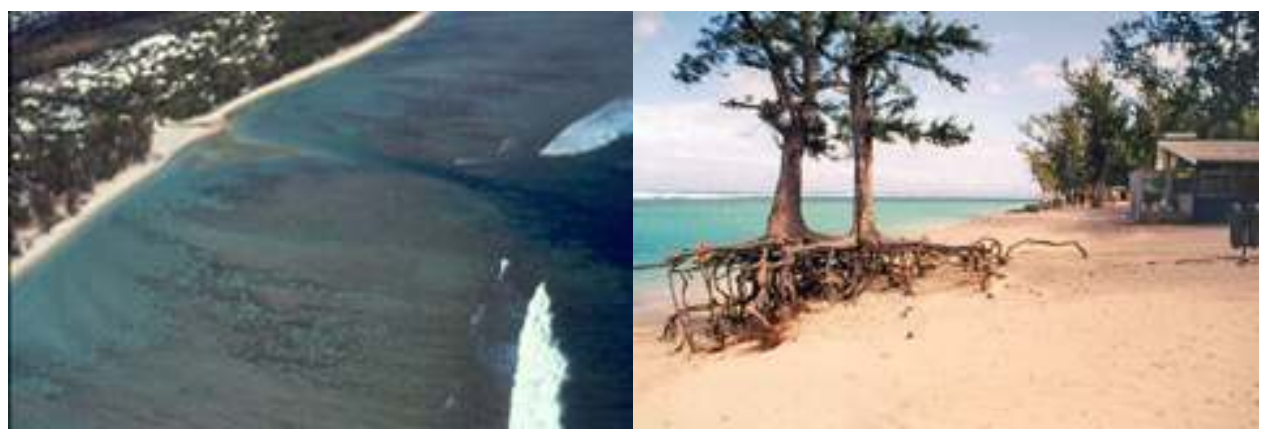

Cette plage étroite (10 m en moyenne) est très affectée par l'érosion en dépit de sa position à l'arrière d'un récif frangeant (photo de gauche. Elle offre des eaux calmes favorables à la baignade et quelques services (bars, restaurants, location de matériel nautique, poste de secours). On y dénombre plus de 4000 personnes au moment des pics de fréquentation. Elle est très accessible et bordée d'aires de stationnement.

[Tous les clichés sont de l'auteur]

\section{Qualité des plages et stratégies de développement touristique}

L'échantillon étudié montre que les facteurs naturels et anthropiques jouent un rôle variable dans la qualité des plages. Parmi les plus attractives, certaines présentent des qualités naturelles exceptionnelles alors que d'autres ont pour principal atout un haut niveau d'équipement et d'entretien. Cette situation traduit, non seulement la 
variabilité des conditions naturelles dans l'espace à différentes échelles, régionale comme locale, mais aussi des différences très importantes entre les dispositifs de gestion, plus ou moins élaborés et interventionnistes. Ceux-ci renvoient à leur tour aux stratégies de développement de ces États et départements insulaires, donc à des choix politiques et économiques.

Par ailleurs, des plages qui ont fait l'objet de travaux d'aménagement paysager importants et qui sont entretenues par les services techniques des hôtels présentent un taux d'attractivité " naturelle » - liée à la qualité du sable, de la végétation et de la zone de bain - élevé. Cela interroge sur la part de nature des éléments dits «naturels » et invite à questionner la relation qui existe entre la naturalité des sites et leur qualité. L'on peut alors se demander dans quelle mesure des interventions humaines peuvent améliorer la qualité de l'environnement littoral et accroître l'attractivité des plages. Dans ces conditions, quel est le poids véritable des attributs dits naturels dans l'attractivité des littoraux tropicaux?

Pour répondre à ces questions, l'on abordera successivement la situation des îles étudiées.

\section{Aux Seychelles, « le luxe à l'état brut »?}

37 Ce slogan touristique du début des années 2000, accompagné de photographies en noir et blanc, donne bien le ton de la stratégie qui a été adoptée par le gouvernement des Seychelles. Le pouvoir d'attraction des paysages et plus globalement de la nature, présentée comme étant préservée et généreuse, constitue depuis le début des années 1970 le fondement de la politique de développement touristique (Gay, 2004 ; Duvat, à paraître). La charge mythique que les plages seychelloises ont acquise à travers le temps, qui repose sur des attributs naturels exceptionnels - une végétation côtière foisonnante, des eaux turquoises, chaudes et calmes, un sable blanc très fin, des chaos de granite très pittoresques - se retrouve dans les motivations des touristes à venir dans cet archipel et dans leur appréciation de la qualité des plages. Leur séjour doit en effet répondre à une attente spécifique: voir ces côtes de granite qui sont mondialement connues. Dans cet archipel, le faible taux de retour des touristes ne s'explique pas seulement par la cherté du séjour et par la médiocrité des distractions qui sont proposées. Elle tient aussi au désir d' " y aller au moins une fois », qui explique le nombre important de voyages de noces qui y sont effectués. Les Seychelles ont acquis la réputation de "paradis de la nature " grâce à la promotion d'espèces végétales, comme le coco-fesse (Lodoicea maldivica), et animales, comme les tortues et les oiseaux marins, et de lieux emblématiques (l'atoll d'Aldabra et la vallée de Mai à Praslin, classés patrimoine mondial de l'humanité au début des années 1980). Cette réputation joue un rôle majeur dans la venue des touristes. De la même manière, les études qui sont réalisées dans des parcs nationaux soulignent le rôle du capital naturel et du paysage dans les motivations des visiteurs et dans leur appréciation des sites (Deng et al., 2002).

Cela explique le taux d'attractivité environnementale très élevé d'un certain nombre de plages qui cumulent nombre de qualités naturelles (fig. 1). Là où les conditions physiques ne sont pas favorables à la baignade, l'attractivité des plages est moins élevée, car ces sites ne font l'objet d'aucune intervention technique (création d'une zone de bain artificielle, par ex.), ni humaine (surveillance de la baignade) visant à 
réduire les contraintes physiques. C'est une nature à l'état brut, non transformée, qui est offerte au visiteur.

Figure 1 - La figure de la plage mythique : Anse Lazio à Praslin

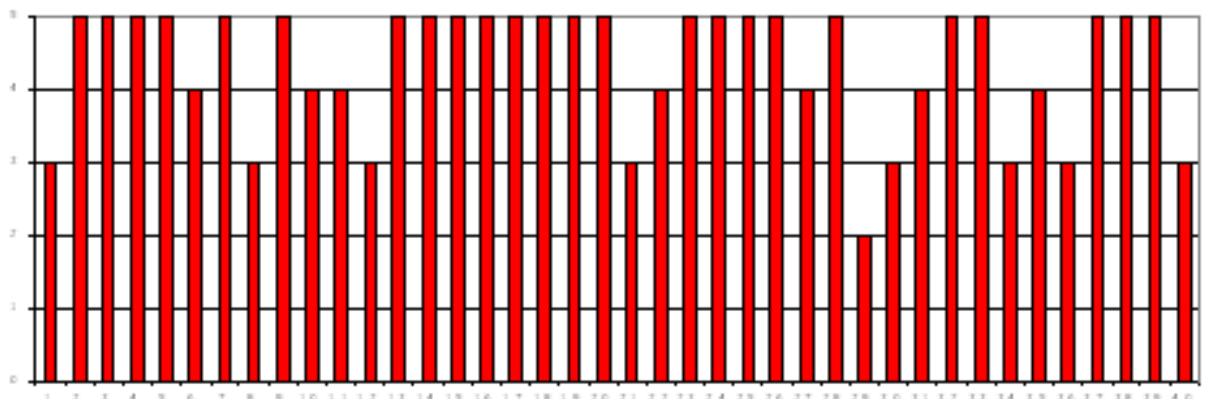

Cette plage est l'une des plus connues des Seychelles. Elle présente d'importants atouts naturels et illustre le caractère très limité de l'équipement.

D’un autre côté, l'attractivité associée aux facteurs humains (équipements, services, accessibilité...) est réduite pour la plupart des plages, à l'exception de celles des grands hôtels. Cela s'explique par deux facteurs, la faiblesse de la demande nationale - le tourisme local est réduit et les pratiques balnéaires restent peu développées - et la stagnation du tourisme depuis une vingtaine d'années. Cette situation originale pour un petit État insulaire dont le développement repose sur le tourisme est due à des raisons politiques, le socialisme ayant limité les investissements des grands groupes touristiques internationaux (Gay, 2004). La rareté des commerces et le faible développement des services (bar, restauration, clubs d'activités) sur le littoral comme dans la capitale tient aux mêmes raisons politiques qui ont façonné un style de vie local non occidental.

40 L'évaluation de la qualité des plages traduit donc bien dans ces conditions « la nature à l'état brut ». Les résultats sont très fortement influencés par les qualités naturelles des sites, qui ont été peu modifiées par l'homme.

\section{À lî̀le Maurice, « des plages quatre étoiles »}

La situation de l'île Maurice est très différente. Les informations recueillies auprès des représentants de l'Association des restaurateurs et hôteliers de l'île Maurice (ARHIM), qui regroupe environ les deux tiers des professionnels, traduisent bien la stratégie nationale : développer le tourisme haut de gamme sur la base de la qualité du service.

Les résultats de notre évaluation retranscrivent bien la politique de qualité que conduisent les compagnies hôtelières. La qualité des plages d'hôtels ne tient pas seulement au fait que ces établissements ont en général été implantés dans des secteurs propices au tourisme. Elle s'explique aussi, d'une part, par les interventions techniques et les opérations d'aménagement qui sont réalisées, et d'autre part, par la priorité qui est accordée à l'équipement. De nombreux sites ont fait l'objet de grands travaux d'aménagement au moment de la construction des hôtels. Des marécages ont été asséchés (Flic en Flac), des dunes aplanies (à Flic en Flac et au Morne, par ex.) et de vastes jardins tropicaux créés. De nombreuses plages ont été débarrassées de leurs éléments grossiers et, sur certaines d'entre elles, les beachrocks ont même été enlevés. 
Le ratissage quotidien des estrans permet d'entretenir la qualité du sable, les débris de corail et les coquillages étant ramassés au même titre que les déchets d'origine humaine. Certaines de ces plages sont périodiquement rechargées en sable et reprofilées à l'aide de petits engins, comme on peut l'observer à la pointe de Flacq au devant de l'hôtel Saint-Géran. Sur les différentes façades littorales, il existe des hôtels dont les plages sont artificielles. Différentes interventions ont également été réalisées dans la zone marine. Là où la faible profondeur du platier récifal limitait les possibilités de baignade et de navigation, des zones de bain ont été creusées et des chenaux de navigation ouverts. Des passes ${ }^{10}$ ont été élargies pour permettre aux bateaux de plaisance d'atteindre les eaux du large (Flic en Flac). Bien peu de plages d'hôtels ont finalement conservé leurs caractéristiques naturelles. La "nature» littorale a été largement artificialisée. La maîtrise que les compagnies hôtelières ont acquise dans la gestion des problèmes environnementaux est illustrée par leurs actions dans le domaine de l'érosion côtière. Les solutions légères (rechargement en sable, installation de sacs de sable) qu'elles pratiquent améliorent l'état des plages alors que le Ministère de l'environnement continue à implanter sur les plages publiques des kilomètres de gabions qui les dénaturent (Cazes-Duvat, 2005). En second lieu, la qualité des plages d'hôtels tient à leur excellent niveau d'équipement. Aux équipements de plage (chaises longues, parasols, toilettes, douches, bars), s'ajoutent divers clubs d'activités, en particulier nautiques, qui offrent au touriste un niveau de confort et une capacité récréative très satisfaisants. C'est le cumul de ces différents facteurs qui explique que les taux d'attractivité les plus élevés se rencontrent à l'île Maurice (fig. 2).

Figure 2 - La qualité des plages d'hôtels à Maurice : l'exemple de Trou aux Biches

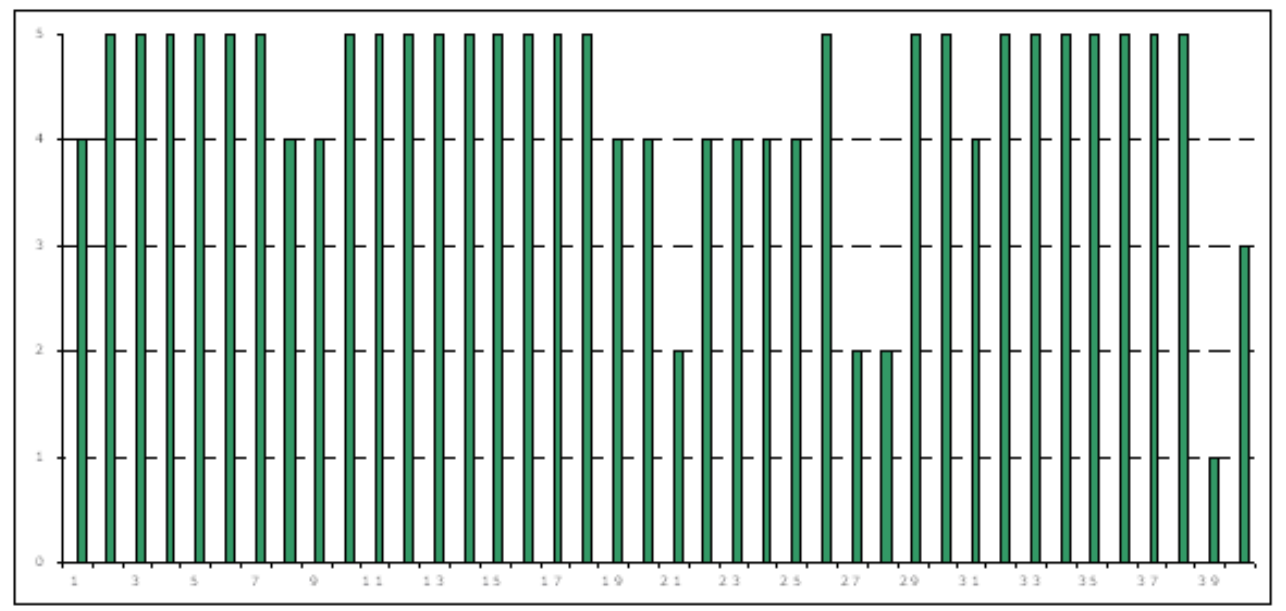

Ces plages présentent d'importants atouts naturels et un haut niveau d'équipement et de service. Les scores les plus faibles traduisent les conséquences du phénomène d'enclavement des hôtels.

Le niveau de qualité relativement élevé des plages publiques traduit un autre aspect de la politique de gestion du littoral, l'effort du gouvernement pour satisfaire la demande des Mauriciens ${ }^{11}$. Après deux décennies de croissance touristique rapide qui ont vu les hôtels de plage se multiplier (1970-1990), les autorités ont classé "publiques" de nombreuses plages afin de les soustraire à la convoitise des compagnies hôtelières. On comptait 20 plages publiques couvrant une surface de 77 ha à la fin des années 1980 . À la suite du classement de 52 plages en 1991 pour un total de 66 plages publiques supplémentaires au cours de cette décennie, la surface de ces plages a atteint $272 \mathrm{ha}$, 
répartis entre les façades nord, est et ouest de l'île (Magnan, 2007). Si certaines sont composées de galets, situées dans des secteurs vaseux ou peu propices à la baignade, d'autres présentent des qualités naturelles tout à fait exceptionnelles, comme celles de la Cambuse, de Pomponnette, de Mon Choisy ou de Belle Mare (fig. 3). Les efforts publics qui sont consentis pour l'entretien et l'équipement de ces plages, mission confiée à la Beach Authority, vont contribuer à réduire l'écart qui demeure entre plages publiques et plages d'hôtels.

Figure 3 - Des plages publiques attractives : l'exemple de Pomponnette à l'île Maurice

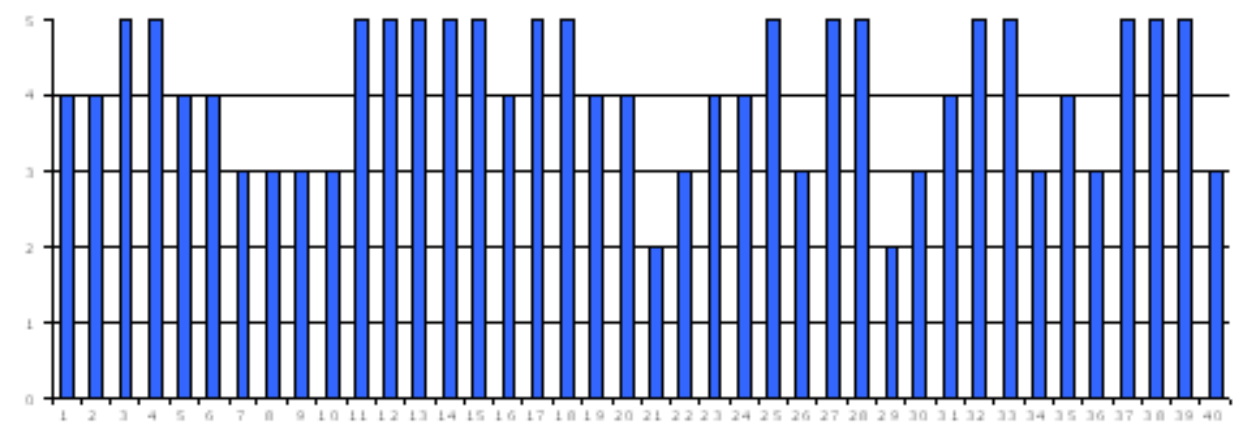

Ce type de plage possède de réelles qualités naturelles. Le niveau d'aménagement et d'équipement reste rudimentaire.

Comment expliquer les effets vertueux du tourisme sur la qualité des plages publiques ? Cet état de fait est-il le reflet d'une politique sociale éclairée ou traduit-il l'existence de tensions qui imposent aux autorités de prendre en compte les aspirations de la société aux loisirs? Quelques exemples illustrent l'ambiguïté de la situation mauricienne qui laisse paraître, au-delà du succès du tourisme et de son acceptation globale par la population, l'existence de réels conflits d'usage. Dans le nord où le tourisme s'est développé dès les années 1970, il reste pour le public peu de plages propices aux usages balnéaires. C'est pour résoudre un conflit - dû à l'utilisation de sa plage par des Mauriciens - que l'hôtel Royal Palm (groupe Beachcomber) a financé l'aménagement de la plage publique voisine de la Cuvette... avant d'édifier un enrochement massif visant à empêcher le public d'atteindre sa plage. Le même type de stratégie a été choisi par les gestionnaires de l'hôtel Legends pour réduire les tensions avec les pêcheurs dont l'accès au littoral a été remis en cause par la construction de l'hôtel. Dans le sud, la dernière région à être mise en tourisme, des compensations financières ont dû être accordées aux pêcheurs afin de les dédommager des impacts de l'implantation de grands hôtels. Ces exemples montrent bien que la politique de protection et d'équipement des plages publiques que conduit le gouvernement relève au moins en partie d'une logique de compensation qui vise à faire accepter à la population les impacts négatifs du tourisme.

\section{À la Réunion, entre politique de rattrapage et nouvelle stratégie de développement touristique}

La politique de gestion du littoral de la Réunion vise au moins trois objectifs : (1) mettre en œuvre la Loi littoral de 1986 et, à ce titre, limiter l'urbanisation et les occupations illégales du domaine public maritime; (2) protéger les récifs coralliens des 
dégradations d'origine anthropique ; (3) améliorer la qualité d'une offre touristique en pleine évolution. D'un côté, il s'agit de contrôler l'urbanisation particulièrement rapide qui s'observe sur la côte corallienne, due à la forte croissance de la population ${ }^{12}$ et à l'attractivité de cette région (zone industrialo-portuaire, bonne accessibilité de SaintDenis par voie rapide, climat favorable...). De l'autre, les collectivités locales s'emploient à promouvoir le développement économique sur la base d'un tourisme de qualité à destination de la clientèle extérieure ${ }^{13}$ et à répondre à la demande des résidents en espaces de tourisme et de loisir. Dans cette société en voie d'occidentalisation rapide, l'augmentation du niveau de vie alimente d'importants flux de tourisme et de loisir vers les littoraux. Or les plages coralliennes de la Réunion ne possèdent pas les qualités de celles de Maurice ou des Seychelles : la plupart d'entre elles sont tapissées de débris et de blocs de corail et bordées, comme on l'a vu, de platiers récifaux affleurants.

$\mathrm{Au}$ cours des dernières années, d'importantes opérations d'aménagement et d'embellissement ont été menées par les communes (Saint-Paul, Saint-Leu, Étang-Salé et Saint-Pierre). Elles ont pris des formes variées: la fermeture de routes côtières (Étang-Salé), l'implantation de rondavelles pour loger les snacks-bars, le remplacement des postes de secours, l'installation sur les fronts de mer d'un système d'éclairage et de plots visant à mettre fin au stationnement sauvage... Dans le cadre d'un partenariat avec l'Université de la Réunion et le parc marin, la SEM balnéaire de Saint-Gilles (commune de Saint-Paul) procède à l'enfouissement des débris de corail dans le corps des plages afin de limiter la gêne qu'ils occasionnent. C'est la seule intervention directe sur la qualité de l'environnement littoral. Elle ne suffit néanmoins pas à donner à la Réunion une qualité de sable égale à celle que l'on trouve à Maurice et aux Seychelles. Par ailleurs, les conditions de baignade restent médiocres à moyennes sur nombre de plages. Les efforts qui sont consentis permettent au total d'offrir des plages de qualité tout à fait correcte (fig. 4).

Figure 4 - Une plage type de la Réunion : I'Ermitage (Saint-Paul)

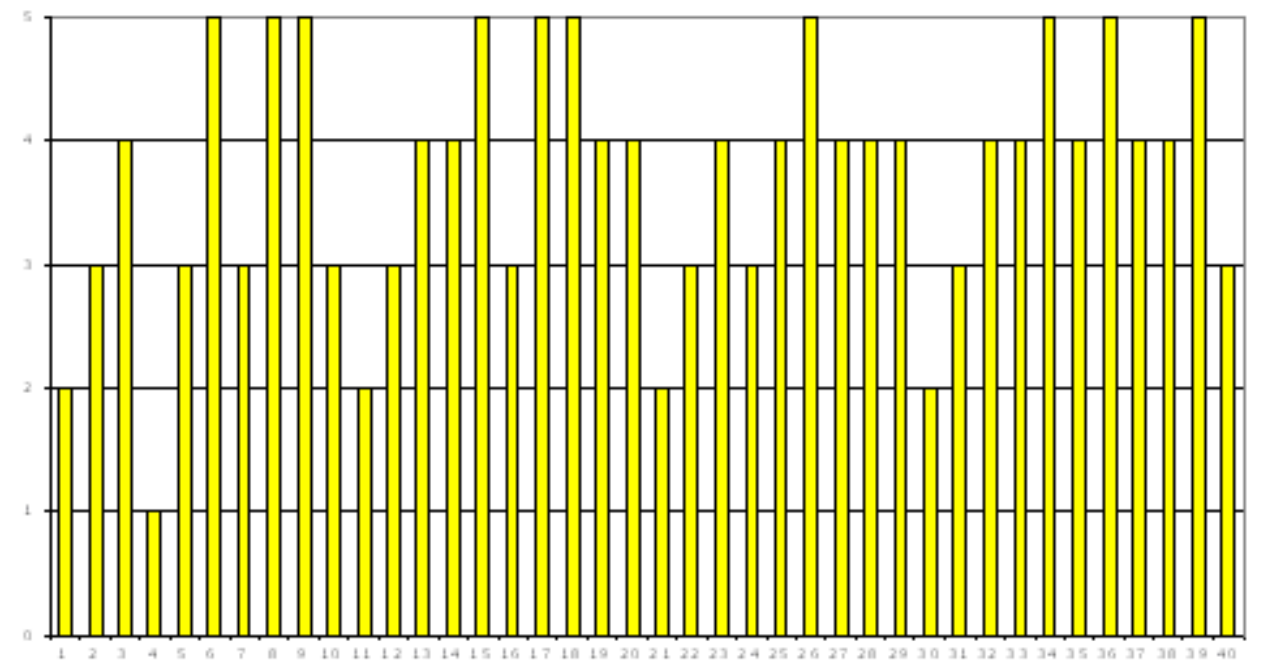

Les attributs naturels sont moins favorables aux pratiques balnéaires que sur la majorité des plages mauriciennes et seychelloises. Le niveau de service est inférieur à celui que l'on trouve à Maurice. 
47 À la différence de que l'on observe à l'île Maurice, le rôle des hôteliers est assez discret. Si quelques hôtels et plagistes proposent des chaises longues et des parasols ou la location d'engins nautiques à leurs clients, et procèdent à un léger ratissage des plages, cette situation reste marginale. Globalement, les acteurs privés influent peu sur la qualité des plages.

48 Les caractéristiques naturelles défavorables que présentent certaines portions $d u$ littoral et le niveau d'équipement relativement faible des plages expliquent que l'on obtienne des taux d'attractivité relativement peu élevés et les valeurs planchers de cette évaluation.

C'est bien parce que les principaux atouts touristiques de la Réunion ne sont pas sur son littoral que la stratégie touristique se réoriente aujourd'hui vers la valorisation du capital naturel des cirques et du volcan, récemment labellisés par la création d'un parc national. La démarche de classement des Hauts de l'île en tant que Patrimoine mondial de l'UNESCO renforcerait cette orientation si elle aboutissait. Dans ces conditions, alors que le tourisme reste identifié comme le moteur du développement, l'on comprend que la priorité des pouvoirs publics soit aujourd'hui de protéger les récifs coralliens des dégradations d'origine anthropique (pêche, piétinement et prélèvements, pollutions diverses). La création d'une réserve marine en février 2007 traduit bien cette préoccupation. Si les slogans touristiques ont radicalement changé avec le passage, en trente ans, de la promotion du littoral à celle de l'« île intense ", il n'en reste pas moins que $67 \%$ des nuitées touristiques sont encore comptabilisées sur la côte corallienne, ce qui rend compte à la fois de la concentration de la capacité d'hébergement dans ce secteur et du souhait des touristes de pouvoir séjourner au moins quelques jours à proximité des plages. Dans ce contexte, la politique de gestion et de protection du littoral qui se développe actuellement traduit d'une part, une volonté d'alignement sur la situation de la Métropole (protection des milieux et mise en application de la réglementation), et d'autre part, une situation économique très particulière, celle d'un DOM dont l'ambition touristique n'est pas comparable avec celle des États insulaires voisins.

\section{Conclusion}

50 Cette étude a d'abord permis de mettre en évidence les effets très contrastés des décennies d'urbanisation et de développement économique sur la qualité de l'environnement littoral. Alors que les impacts négatifs de la littoralisation sont couramment soulignés, il semble important de dresser des diagnostics objectifs et plus complets. Par exemple, aux Seychelles où les effectifs touristiques stagnent autour de 120000 visiteurs et où a été fait le choix du tourisme haut de gamme, les effets dégradants des aménagements sont limités et ils ne sont en général pas dus au tourisme. Les attributs naturels exceptionnels des îles granitiques, qui constituent le fondement de la stratégie de développement touristique, sont largement préservés. Très différent, le cas mauricien montre que l'attractivité des plages, telle qu'elle est perçue par les touristes, repose sur une forte artificialisation des sites, ce qui démontre que des interventions humaines peuvent améliorer la qualité de l'environnement.

51 Ce constat soulève la question du rôle des attributs naturels dans l'attractivité des plages de la zone intertropicale. Si le caractère à la fois exceptionnel et unique des 
paysages seychellois constitue le principal atout touristique de cet archipel, cette situation reste marginale. Le succès de la stratégie mauricienne fondée sur un haut niveau de service met en évidence, d'une part, le caractère attractif de sites très artificialisés dont les qualités «naturelles » ont été améliorées ou produites, et d'autre part, le rôle majeur des équipements, du confort et de la capacité récréative pour les visiteurs qui fréquentent ce type de destination.

Cette étude montre également que la qualité des plages s'inscrit au cœur des stratégies de développement des petites îles tropicales, et en particulier des petits États en développement. D'abord, parce qu'elle est fortement intégrée aux stratégies touristiques. Ensuite, parce qu'elle témoigne du rôle variable des acteurs publics (Seychelles, Réunion) et privés (Maurice) d'un pays à un autre. Enfin, parce que les politiques de gestion du littoral - on retiendra l'exemple des plages publiques à Maurice - témoignent des enjeux sociétaux et des tensions sociales qui se font jour après quatre décennies de développement. L'urbanisation rapide et la diffusion du modèle de consommation et de loisirs de l'Occident créent de nouveaux enjeux sur le littoral, qui sont relatifs à la fois à sa qualité (augmentation des exigences des populations locales) et à son partage (hôtels/public).

Ce type d'outil peut être utile aux gestionnaires, publics et privés, en leur permettant de disposer de tableaux de bord pour réaliser des suivis de la qualité du littoral et des effets, environnementaux et sociaux, des politiques qu'ils mettent en œuvre. L'association de relevés de terrain et d'enquêtes qu'implique ce type de méthodologie constitue une richesse, car elle permet de dresser un diagnostic complet (données d'état et de perception). Une première expérimentation de ce type d'outil a été lancée à la Réunion. Elle consiste en un guide d'entretien des plages qui a été produit en partenariat avec la SEM balnéaire de Saint-Gilles afin que le travail de terrain qu'effectuent les techniciens puisse être évalué (Aimé, 2005).

\section{BIBLIOGRAPHIE}

Aimé D., 2005, Gestion du littoral : mieux communiquer pour mieux préserver. Un manuel de procédures pour la SEM Station Balnéaire de Saint-Gilles (Saint-Paul, la Réunion). Mémoire de Master Professionnel, Sciences et gestion de l'environnement tropical, Université de la Réunion, 107 p. + manuel.

Cazes G., 1989, L'île tropicale, figure emblématique du tourisme international », dans Iles et tourisme en milieux tropical et subtropical, Actes du XIe colloque de la SEPANRIT, Talence, Collection Iles et Archipels n 10, 1989, p. 37-53.

Cazes-Duvat V., 1999, Les littoraux des îles Seychelles, Paris, l'Harmattan, 366 p . 7 cartes couleur hors-texte.

Cazes-Duvat V. (avec la participation de R. Robert), 2001, Atlas de l'environnement côtier des îles granitiques de l'archipel des Seychelles, Université de La Réunion/Région Réunion/CIRAD Montpellier, Louis Jean, Gap, 96 pages couleur. 
Cazes-Duvat V., 2005, Les archipels de l'ouest de l'océan Indien face à l'érosion côtière (Mascareignes, Seychelles, Maldives), Les Annales de Géographie, 644, p. 342-361.

Cazes-Duvat V., Paskoff R., 2004, Les littoraux des Mascareignes entre nature et aménagement. Paris, L'Harmattan, 187 p. +16 planches couleur.

Cazes-Duvat V., Pesme J.-O., 2002, Étude de capacité de charge des plages des côtes ouest et sud de l'île de la Réunion. Rapport de synthèse. Étude réalisée pour le compte de la Région Réunion, $63 \mathrm{p}$.

Cendrero A., Fischer D.W., 1997, A procedure for assessing the environmental quality of coastal areas for planning and management. Journal of Coastal Research, 13(3), p. 732-744.

Cendrero A., Francés E., Del Corral D., Ferman J.L., Fischer D., del Rio L., Camino M., Lopez A., 2003, Indicators and indices of environmental quality for sustainability assessment in coastal areas : application to case studies in Europe and America. Journal of Coastal Research, 19(4), p. 919-933.

Cervantes O., Espejel H., 2008, Design of an integrated evaluation index for recreationla beaches. Ocean and Coastal Management, 51, p. 410-419.

Deng J., King B., Bauer T., 2002, Evaluationg natural attractions for tourism. Annals of Tourism Research, 29(2), p. 422-438.

Duvat V., 2007, Les littoraux coralliens des petites îles de l'océan Indien (Mascareignes, Seychelles, Maldives). Volume 2, Aménagement et gestion. Paris, Institut Océanographique, Collection Océanis , fascicule $n^{\circ} 31-3 / 4$ (p. 189-334).

Duvat V., 2008, L'intérêt d'une approche en termes de capacité de charge pour les gestionnaires : l'exemple de l'île de La Réunion (océan Indien). Colloque International pluridisciplinaire « Les littoraux : subir, dire, agir », Lille, 16-18 janvier 2008.

Duvat V., à paraître, Mythes et réalités de la nature en milieu tropical insulaire : l'exemple de l'archipel des Seychelles. In : Insularités et insularismes d'hier et d'aujourd'hui. Hommage au professeur Christian Huetz de Lemps, Paris, Presses Universitaires de Paris Sorbonne, 15 p. environ.

Ergin A., Micallef A., Williams A., 2008, Coastal scenic evaluation of Gozzo/Gomino, Malta, as a tourism product, Colombo. Colloque International pluridisciplinaire « Les littoraux : subir, dire, agir », Lille, 16-18 janvier 2008

Ethos Consulting, 1991, Natural resource based tourism in northwest British Columbia. Vancouver, Ministry of Development, Trade and Tourism.

Ferrarini A., Bodini A., Becchi M., 2001, Environmental quality and sustainability in the province of Reggio Emilia (Italy) : using multi-criterai analysis to assess and compare municipal performance. Journal of Environmental Management, 63, p. 117-131.

Fines K.D., 1968, Landscape evaluation : a research project in East Sussex. Regional Studies, 2, p. 41-55.

Gay, J.-Ch., 2004, Tourisme, politique et environnement aux Seychelles. Tiers-Monde, TXLV, 178, p. 319-339.

Kaplan R., Kaplan S., 1989, The visual environment : public participation in design and planning. Journal of Social Issues, 45, p. 59-86.

Klein J., 2003, Protéger le littoral dans les départements français d'Outre-Mer. Thèse de doctorat de géographie, Université de Paris IV Sorbonne, 545 p. 
Leatherman S., 1997, Beach rating : a methodological approach. Journal of Coastal Research, 13(1), p. 253-258.

Magnan A., 2005, Tourisme, développement et dynamique territoriale dans l'archipel des Maldives et à l'île Maurice (océan Indien). Thèse de doctorat de géographie, Université de Montpellier III, $462 \mathrm{p}$.

Magnan A., 2007, Tourisme et réserves d'espace pour les pratiques locales sur les littoraux de l'île Maurice, Les Cahiers d'Outre-Mer, 240, p. 341-372.

Micallef A., 2002, Bathing area management in the Maltese islands. PHD Thesis, University of Wales, $541 \mathrm{p}$.

Micallef A., Williams A.T., Radic M., Ergin A., 2004, A novel bathing area registration evaluation system : the dalmatian (Croatia) coast. Jn. World Leisure, 46(4), p. 4-21.

Morgan R., 1999, A novel, user-based rating system for toourist beaches. Tourism Management, 20, p. 393-410.

Morgan R., Williams A.T., 1995, Socio-demographic parameters and user priorities at Gower beaches, UK. In : Healy M.G. \& Doody J.P. (eds), Directions in European Coastal Management. Cardigan, Wales, UK, EUCC \& Samara Publishing Ltd, p. 83-90.

Moya J.-Ph., 2000, Gestion de la fréquentation des plages : la problématique des accès à la mer. Application à la côte ouest de l'île de la Réunion. Mémoire de maîtrise de géographie, Université de la Réunion, $129 \mathrm{p}$.

Nelson C., Morgan R., Williams A.T., Wood J., 2000, Beach awards and management. Ocean and Coastal Management, 43, p. 87-98.

Pereira L.C.C., Jimenez J.A., Medeiros C., Marinho da Costa R., 2003, The influence of the environmental status of Casa Caiada and Rio Doce beaches (NE Brazil) on beaches users. Ocean and Coastal Management, 46, p. 1011-1030.

Virgin Islands Planning Office, 1977, Preliminary program Virgin Islands Coastal Zone Management, Charlotte Amalie, Iles Vierges Américaines, 194 p.

Williams A.T., Morgan R., 1995, Beach awards and rating systems, Shore and Beach, 63(4), p. 29-33.

Yang G., 1994, Tourism resources, Kunming, Yunnan University Press.

Zube E.H., Pitt D.G., 1981, Cross-cultural perceptions of scenic and heritage landscapes. Landscape planning, 8, p. 69-87.

\section{NOTES}

1. La situation de l'île Maurice en donne une excellente illustration, avec l'adoption de plans d'orientations stratégiques et de lois qui font de la qualité du littoral une priorité nationale (National Environment Action Plan de 1991 et National Environment Strategy de 1999).

2. La réalisation d'une étude d'accessibilité des plages sur la côte corallienne de la Réunion (Moya, 1999) a montré que l'urbanisation côtière s'était accompagnée de l'appropriation de nombreux chemins d'accès aux plages par les riverains et que les servitudes de passage n'étaient pas respectées (fermeture des accès et mise en œuvre de mesures dissuasives à l'encontre du public). 
3. Par le local governement act (1989), ce ministère a acquis la compétence de déclarer publiques un certain nombre de plages. On en compte aujourd'hui 87, qui représentent une surface de 272 ha.

4. Son périmètre d'action est la principale commune du littoral corallien, celle de Saint-Paul.

5. La réalisation de comptages à la Réunion et à Maurice entre 1998 et 2004 a montré une augmentation régulière de la fréquentation des plages (Cazes-Duvat et Pesme, 2002 ; Magnan, 2007).

6. Les plages de sable noir sont moins prisées que celles de sable blanc, notamment parce qu'elles sont brûlantes en saison chaude.

7. Elle est approximativement de $25 \mathrm{~km}$ à la Réunion, de $220 \mathrm{~km}$ à l'île Maurice, de $36 \mathrm{~km}$ à Mahé, de $21 \mathrm{~km}$ à Praslin et de $8 \mathrm{~km}$ à la Digue, si l'on ne retient que ces îles.

8. Il est proposé au lecteur de consulter les ouvrages disponibles pour localiser ces plages (CazesDuvat, 2001, p.78-79 ; Cazes-Duvat et Paskoff, 2004, p. 28 et 170).

9. Le beachrock ou grès de plage est une formation rocheuse correspondant à une ancienne plage qui s'est cimentée. Il se présente souvent sous la forme d'une ou de plusieurs dalles inclinées vers la mer et situées dans la zone intertidale. Lorsqu'elles sont massives, elles nuisent à l'exercice d'activités balnéaires sur la plage comme dans les eaux côtières.

10. Les passes sont des ouvertures dans les récifs coralliens qui correspondent au prolongement sous le niveau de la mer de vallées continentales. Elles se sont formées lors de régressions marines.

11. Le développement soutenu et rapide des hôtels a eu pour effet une privatisation de fait d'importantes portions du littoral sableux, qui sont devenues inaccessibles au public en raison du contrôle des accès aux hôtels et à leurs plages (Magnan, 2007).

12. La population de la Réunion devrait passer des quelque 740000 habitants actuels à plus d'un million autour de 2020.

13. La création du parc naturel national de la Réunion, qui intègre le volcan, les cirques et les forêts primaires, constitue un élément clé du renouvellement de la politique de promotion touristique et en particulier de la valorisation des Hauts. Mais les touristes souhaitent néanmoins

\section{RÉSUMÉS}

La qualité des plages s'inscrit au cœur des enjeux de développement des petites îles tropicales en raison d'une part, de la place qu'elle occupe dans les stratégies de promotion touristique, et d'autre part, de l'importance que prend le littoral dans les pratiques récréatives et touristiques émergentes des populations locales. Dans ce contexte, l'objectif de cet article est de présenter les résultats d'une évaluation réalisée dans différentes îles des archipels des Seychelles et des Mascareignes, et de mettre en évidence la relation qui existe entre la qualité des plages, leurs attributs naturels et les politiques d'aménagement et de gestion des acteurs publics et privés.

Today, the quality of the tropical beaches is closely linked to development challenges for two main reasons. At first, because sandy beaches play an important role in tourism development policies, and secondly because of the emergence of the touristic and recreative activities of the local communities. In this context, the purpose of this paper is to expose the results of an assessment which was done in the Seychelles and Mascarene islands and to highlight the 
relationship which can be observed between beach quality, natural assets and the strategies of public and private actors.

\section{INDEX}

Mots-clés : qualité des plages, environnement littoral, tourisme, îles tropicales, océan Indien Keywords : beach quality, coastal environment, tourism, tropical islands, Indian ocean

\section{AUTEUR}

\section{VIRGINIE DUVAT}

Virginie Duvat (virginie.magnan@univ-lr.fr) est professeur de géographie à l'Université de la Rochelle et membre de l'UMR CNRS LIENSs-6250. Elle a récemment publié un ouvrage en deux volumes intitulé Les littoraux coralliens des petites îles de l'océan Indien (Mascareignes, Seychelles, Maldives), Paris, Institut Océanographique, Collection Océanis, fascicules n³1-1/2 (189 p) et $n^{\circ} 31-3 / 4$ (p. 189-334) - qui traite des aspects géomorphologiques (vol. 1) et des questions liées à l'aménagement et à la gestion (vol. 2) ainsi que deux articles intitulés « Mondialisation touristique et environnement dans les petites îles tropicales ", Les Cahiers d'Outre-Mer, 236 p. 513-539 et « Proposition de typologie des plages coralliennes (océan Indien occidental) », Zeitschrift für Geomorphologie. 51(3) p. 307-325. 